\title{
On Pareto Efficiency in Asset Markets
}

\author{
Kazuhiro Takino \\ Graduate School of Management, Nagoya University of Commerce and Business, Nagoya, Japan \\ Email: takino@nucba.ac.jp
}

How to cite this paper: Takino, K. (2019) On Pareto Efficiency in Asset Markets. Theoretical Economics Letters, 9, 2508-2515. https://doi.org/10.4236/tel.2019.97158

Received: August 8, 2019

Accepted: October 8, 2019

Published: October 11, 2019

Copyright @ 2019 by author(s) and Scientific Research Publishing Inc. This work is licensed under the Creative Commons Attribution International License (CC BY 4.0).

http://creativecommons.org/licenses/by/4.0/

\begin{abstract}
In this study, we consider Pareto efficiency in financial markets. In welfare economics, it is sufficient to consider competitive equilibrium to assure $\mathrm{Pa}$ reto efficiency. This study, however, focuses on describing the utility possibility frontier, which explicitly shows Pareto efficiency for financial markets. To this end, we use the time-additive utility (functional) with the mean-variance utility. In deriving the utility possibility frontier, we obtain an asset pricing formula dependent on an agent's utility. We provide a characteristic of this formula to ensure Pareto efficiency. Moreover, our study generalizes the payoff function of the asset. This enables us to analyze various financial transactions. As an application of our framework, we consider a simple insurance contract with default. We then show that the likelihood of default makes the market Pareto inefficient or deteriorates social welfare, as shown in previous studies.
\end{abstract}

\section{Keywords}

Pareto Efficiency, Utility Possibility Frontier, Default Risk

\section{Introduction}

In this study, we consider Pareto efficiency in financial markets. Pareto efficiency is a criterion in microeconomics, showing optimality in exchanges of goods, services, and financial assets. We say an exchange is Pareto efficient if an agent cannot increase her/his welfare without decreasing the welfare of others. Previous studies have demonstrated how the regulations in financial transactions and financial instruments (including products) improve Pareto efficiency in the incomplete (financial) markets [1] [2] [3] [4] [5]. The default in financial transaction is the most typical example of the incomplete market. For example, Geanakoplos [3] shows that the introduction of collateral improves Pareto efficiency in the market. Acharya and Bisin [1] and Taddei [5] also consider collateralization in analyzing Pareto efficiency. 
As highlighted in textbooks on microeconomics [6], Pareto efficiency is described using the so-called Utility Possibility Frontier (Figure 1). Figure 1 shows a sample of the utility possibility frontier between two agents. The curve in the figure is right-decreasing. This implies that if one agent wants to increase its utility, the other has to decline. Hence, the points on the utility possibility frontier satisfy Pareto efficiency. Using the utility possibility frontier, Geanakoplos [3] demonstrates that a defaultable economy reduces Pareto efficiency. Therefore, needless to say, the utility possibility frontier is useful and accessible for analyzing the welfare of an economy.

While the Pareto criterion has been used in analyzing financial markets, few studies describe the utility possibility frontier explicitly (we only introduced Geanakoplos [3]). However, as this study reveals, it is necessary to pose some assumptions in the model to explicitly describe the utility possibility frontier. In fact, Geanakoplos [3] has never provided the function of the frontier. In this study, we construct a model to explicitly describe the utility possibility frontier in financial markets. The model includes 1) the utility form 2) the price depending on an agent's utility. We apply the time-additive utility functional with the mean-variance utility function as the utility form. To derive the utility possibility frontier, we obtain a pricing formula that depends on the utility of an agent. We find a characteristic of the utility-depended pricing formula to satisfy Pareto efficiency. Moreover, we try to generalize the asset class while we restrict the number of market participants and their utility forms.

The contribution of this study is to provide a framework to explicitly describe social welfare using the utility frontier curve satisfying Pareto efficiency. Moreover, the payoff function of assets is generalized. This enables us to examine various asset classes, such as derivatives, insurance, and so on. In fact, as a numerical example, we consider a simple insurance contract with default. We then describe

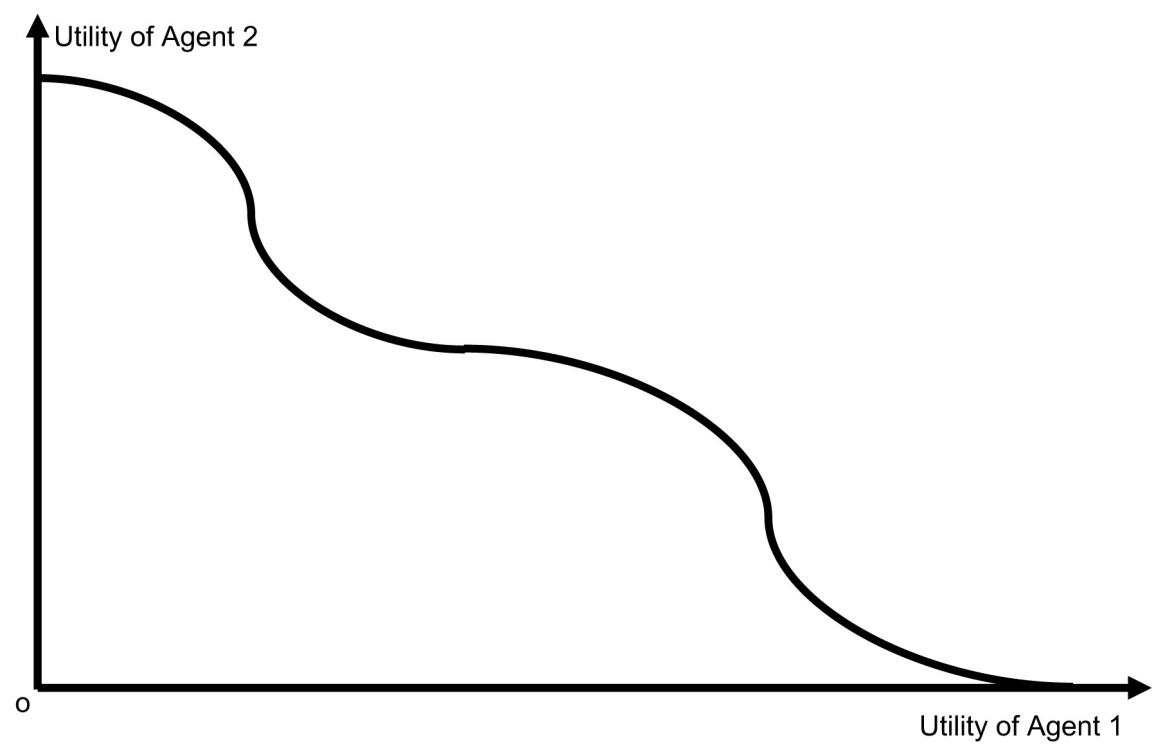

Figure 1. Utility possibility frontier. 
that social welfare under the defaultable setting is lower than under the non-default environment, as shown in Acharya and Bisin [1], and Geanakoplos [3].

The rest of this paper is organized as follows. In the next section, we present an economic model. In Section 3, we consider the utility maximization problems of agents, and describe the utility possibility frontier. In Section 4, we carry out the numerical implementation for a simple insurance contract. Section 5 concludes this study.

\section{Model}

Let us consider a one-period economy. In our economy, two agents consume their endowments at each time $(t=0, T)$ and trade a risky asset at time 0 . We denote the price of the asset at time 0 by $q$, and its value (or payoff) at time $T$ by $g(T)$. Our risky asset includes stock, derivatives, and insurance. For convenience, we assume an agent is a buyer (" $b$ ") of the asset and the other is a seller (" $s$ ") of the asset. We denote $k_{j}(j=b, s)$ for the trading volume of the asset that agent $j$ is willing to have. We suppose that the endowments of both agents at time $T$ are random. If the endowment of an agent is negatively correlated with the asset price (or its payment amount) at time $T$, the agent must have an incentive to trade the asset. Thus, the risky asset plays the role of insurance. In the following numerical example, we consider that the agent purchases an asset as insurance.

We denote the endowment of agent $j(j=b, s)$ at time $t$ by $w_{t}^{j}$, and the consumption of agent $j(j=b, s)$ at time $t$ by $x_{t}^{j}$. Then, the budget constraint of agent $b$ (i.e., the buyer) is as follows:

$$
\begin{aligned}
& x_{0}^{b} \leq w_{0}^{b}-k_{b} q, \\
& x_{T}^{b} \leq w_{T}^{b}+k_{b} g(T) .
\end{aligned}
$$

On the other hand, the budget constraint of agent $s$ (i.e., the seller) is as follows:

$$
\begin{aligned}
& x_{0}^{s} \leq w_{0}^{s}+k_{s} q, \\
& x_{T}^{s} \leq w_{T}^{s}-k_{s} g(T) .
\end{aligned}
$$

\section{Optimization Problem}

We suppose that the utility functions of agents are the so-called time-additive type, and agents determine the trading volume $k$ of the asset to maximize their (expected) utilities, that is, the utility of agent $j(j=b, s)$ is represented by

$$
U_{j}\left(x_{0}^{j}, x_{T}^{j}\right)=u\left(x_{0}^{j}\right)+\mathrm{e}^{-\delta_{j} T} u\left(x_{T}^{j}\right),
$$

where

$$
u_{j}(x)=E[x]-\frac{\gamma_{j}}{2} \operatorname{Var}[x]
$$

for $j=b, s$, and $\gamma_{j}$ is the risk-aversion and $\delta_{j}$ is the time preference of agent $j$. Note that $u_{j}(\cdot)$ is the mean-variance utility, which has been used to describe the optimization problem for economic agents [1] [7] [8] [9]. Although several 
utility functions are applied to the time-additive utility form (c.f., Chapter 6 in Munk [10]), we think that the mean-variance type is useful for explicitly describing the utility possibility frontier.

The optimization problem of agent $b$ is

$$
\begin{array}{ll}
\max _{k_{b}} & U_{b}\left(x_{0}^{b}, x_{T}^{b}\right), \\
\text { s.t. } & x_{0}^{b}=w_{0}^{b}-k_{b} q, \\
& x_{T}^{b}=w_{T}^{b}+k_{b} g(T) .
\end{array}
$$

The problem of agent $s$ is

$$
\begin{array}{ll}
\max _{k_{s}} & U_{s}\left(x_{0}^{s}, x_{T}^{s}\right), \\
\text { s.t. } & x_{0}^{s}=w_{0}^{s}+k_{s} q, \\
& x_{T}^{s}=w_{T}^{s}-k_{s} g(T) .
\end{array}
$$

In the previous section, the budget constraints are represented with inequality. However, as the utility functions of both agents are monotonically increasing with their consumptions, the constraints hold in the equality.

By substituting the budget constraints into objective functions, the above problems are rewritten as

$$
\begin{aligned}
\max _{k_{b}} U_{b}\left(w_{0}^{b}-k_{b} q, w_{T}^{b}+k_{b} g(T)\right) \\
=\max _{k_{b}} u_{b}\left(w_{0}^{b}, w_{T}^{b}\right)-k_{b} q+\mathrm{e}^{-\delta_{b} T}\left(E[g(T)] k_{b}-\frac{\gamma_{b}}{2} \operatorname{Var}[g(T)] k_{b}^{2}\right. \\
\left.\quad-\gamma_{b} \operatorname{Cov}\left[w_{T}^{b}, g(T)\right] k_{b}\right) .
\end{aligned}
$$

The problem of agent $s$ is

$$
\begin{aligned}
& \max _{k_{s}} U_{s}\left(w_{0}^{s}+k_{s} q, w_{T}^{s}-k_{s} g(T)\right) \\
& =\max _{k_{s}} u_{s}\left(w_{0}^{s}, w_{T}^{s}\right)+k_{s} q-\mathrm{e}^{-\delta_{s} T}\left(E[g(T)] k_{s}+\frac{\gamma_{s}}{2} \operatorname{Var}[g(T)] k_{s}^{2}\right. \\
& \left.\quad-\gamma_{s} \operatorname{Cov}\left[w_{T}^{s}, g(T)\right] k_{s}\right) .
\end{aligned}
$$

We set

$$
\begin{aligned}
& \alpha_{j}=\frac{\mathrm{e}^{\delta_{j} T}}{\gamma_{j} \operatorname{Var}[g(T)]}, \\
& \beta_{j}=\frac{E[g(T)]-\gamma_{j} \operatorname{Cov}\left[w_{T}^{j}, g(T)\right]}{\gamma_{j} \operatorname{Var}[g(T)]}
\end{aligned}
$$

for $j=b, s$. Then, the optimization problems (3.1) and (3.2) are given by

$$
\begin{gathered}
\max _{k_{b}} U_{b}\left(w_{0}^{b}-k_{b} q, w_{T}^{b}+k_{b} g(T)\right)=\max _{k_{b}} u_{b}\left(w_{0}^{b}, w_{T}^{b}\right)-k_{b} q-\frac{1}{2 \alpha_{b}} k_{b}^{2}+\frac{\beta_{b}}{\alpha_{b}} k_{b}, \\
\max _{k_{s}} U_{s}\left(w_{0}^{s}+k_{s} q, w_{T}^{s}-k_{s} g(T)\right)=\max _{k_{s}} u_{s}\left(w_{0}^{s}, w_{T}^{s}\right)+k_{s} q-\frac{1}{2 \alpha_{s}} k_{s}^{2}-\frac{\beta_{s}}{\alpha_{s}} k_{s},
\end{gathered}
$$


respectively.

From the first order condition, the optimal trading volumes for the asset are

$$
\begin{gathered}
k_{b}=-\alpha_{b} q+\beta_{b}, \\
k_{s}=\alpha_{s} q-\beta_{s} .
\end{gathered}
$$

While we must have an equilibrium volume and price for the asset from (3.6) and (3.7), as Takino [9], we proceed to consider the condition of Pareto efficiency in deriving the utility possibility frontier.

\section{Utility Possibility Frontier}

If the market is Pareto efficient, the utility possibility frontier is defined by

$$
U_{s}=f\left(U_{b}\right),
$$

satisfies

$$
f^{\prime}<0 \text { or } \frac{\partial U_{s}}{\partial U_{b}}<0 .
$$

To ensure Pareto efficiency, we first consider the optimized utility $U_{b}^{*}$ of agent $b$. By substituting (3.6) into (3.4), we have

$$
U_{b}^{*}=u_{b}\left(w_{0}^{b}, w_{T}^{b}\right)+\frac{1}{2 \alpha_{b}}\left(\alpha_{b} q-\beta_{b}\right)^{2} .
$$

Fixing the utility level of agent $b$ as $U_{b},(3.8)$ is

$$
U_{b}=u_{b}\left(w_{0}^{b}, w_{T}^{b}\right)+\frac{1}{2 \alpha_{b}}\left(\alpha_{b} q-\beta_{b}\right)^{2} .
$$

(3.9) gives

$$
q\left(U_{b}\right)=\frac{\beta_{b} \pm \sqrt{2 \alpha_{b}\left(U_{b}-u_{b}\left(w_{0}^{b}, w_{T}^{b}\right)\right)}}{\alpha_{b}} .
$$

(3.10) is a pricing formula for the asset and depends on the utility of agent $b$. The sign of the square-root in (3.10) might be determined to satisfy Pareto efficiency. Unfortunately, we cannot determine the sign at this stage.

Next, the optimal utility of agent $s$ is given by substituting (3.7) into (3.5). That is,

$$
U_{s}=u_{s}\left(w_{0}^{s}, w_{T}^{s}\right)+\frac{1}{2 \alpha_{s}}\left(\alpha_{s} q-\beta_{s}\right)^{2} .
$$

Substituting (3.10) into (3.11), we have candidates for the utility possibility frontier, that is,

$$
U_{s}=f\left(U_{b}\right):=u_{s}\left(w_{0}^{s}, w_{T}^{s}\right)+\frac{1}{2 \alpha_{s}}\left\{\alpha_{s}\left(\frac{\beta_{b} \pm \sqrt{2 \alpha_{b}\left(U_{b}-u_{b}\left(w_{0}^{b}, w_{T}^{b}\right)\right)}}{\alpha_{b}}\right)-\beta_{s}\right\}^{2} .
$$

In the next section, we numerically implement the utility possibility frontier 
(3.12), and analyze the characteristic of (3.10) to satisfy Pareto efficiency.

\section{Numerical Example}

We consider a simple finite state model based on Acharya and Bisin [1]. The economic state at time $T$ takes two states, that is, "Good" (“ $G$ ") and "Bad" (“ $B$ "). We suppose that the probability of $G$ is $p(0 \leq p \leq 1$, automatically, the probability of $B$ is $1-p$ ). Moreover, we consider a defaultable economy. That is, agent $s$ fails to payout the exact claim value $g(T)$ due to the economic state at time $T$.

The endowment of agent $b$ in state $G$ is $w_{T}^{b}=w^{b}(G)>0$ and that in state $B$ is zero. The endowment of agent $s$ in state $G$ is zero, and that in state $B$ is $w_{T}^{s}=w_{N D}^{s}(B)$ without default and $w_{T}^{s}=w_{D}^{s}(B)$ with default $\left(w_{N D}^{s}(B)>w_{D}^{s}(B) \geq 0\right.$, without loss of generality). The payoff of the contingent claim in state $G$ is zero, and that in state $B$ is $g(T)=R$ without default and $g(T)=R_{D}$ with default $\left(R>w_{D}^{s}(B)>R_{D} \geq 0\right.$, without loss of generality). The amount of $R$ is predetermined between agents; however, agent $s$ cannot exactly pay $R$ when she/he defaults. Note that the assumptions of $w_{N D}^{s}(B)>w_{D}^{s}(B)$ and $R>w_{D}^{s}(B)>R_{D}$ shows why agent $s$ is going to default. We suppose that the default probability of agent $s$ is $p_{D}\left(0 \leq p_{D} \leq 1\right)$. Then, $p_{D}=0$ implies that there is no default in the economy. Furthermore, the asymmetry of agents' endowments at each state motivates them to trade the contingent claim. Hence, the agents effectively trade the contingent claim $g$ as insurance. Table 1 lists the parameter values used in this implementation.

Figure 2 shows the utility possibility frontiers for the transaction of the claim. The curves in the figure are right-decreasing, that is, any point on the curve satisfies Pareto efficiency. From the figure, we observe that the curve of "ND" lies

Table 1. Parameters used in the numerical example. The values are based on Acharya and Bisin (2014).

\begin{tabular}{cl}
\hline Parameter & Value \\
\hline$T$ & 0.25 \\
$w_{0}^{b}$ & 0.50 \\
$w_{0}^{s}$ & 5.00 \\
$w^{b}(G)$ & 5.00 \\
$w_{N D}^{s}(B)$ & 10.00 \\
$w_{D}^{s}(B)$ & 12.00 \\
$R$ & 6.00 \\
$R_{D}$ & 10.00 \\
$\gamma_{b}$ & 6.00 \\
$\gamma_{s}$ & 1.00 \\
$\delta_{b}$ & 1.00 \\
$\delta_{s}$ & 0.00 \\
\hline
\end{tabular}




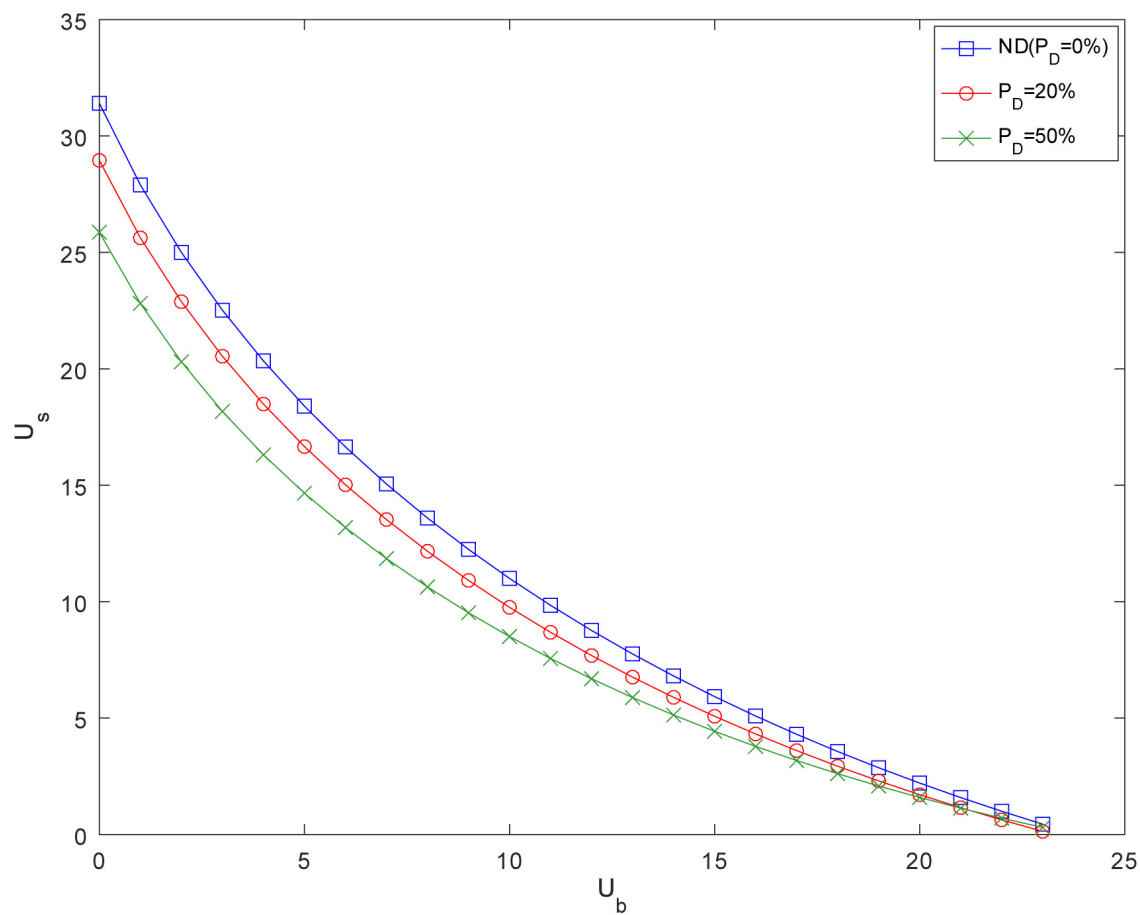

Figure 2. Utility possibility frontiers. "ND" is the non-default case (i.e. $p_{D}=0.00 \%$, marked with “ $\square$ ”), “ $P_{D}=20 \%$ ” (marked with “。”) and “ $P_{D}=50 \%$ ” (marked with “ $\times$ ”) are the defaultable cases. " $P_{D}=20 \%$ " is the case of $p_{D}=0.20$, and " $P_{D}=50 \%$ " is the case of $p_{D}=0.50$. This figure is described by (3.12) with (4.1).

at the top, the curve of the default probability $20 \%$ lies in the middle, and the curve of the default probability $50 \%$ lies at the bottom. This means that the likelihood of default deteriorates social welfare. In other words, any points under the default cases are plotted below the curve of ND. This implies that the transaction of the claim with default is Pareto inefficient, as shown in Acharya and Bisin [1], and Geanakoplos [3]. Finally, the figure is described under the utility-dependent price (3.10) as

$$
q\left(U_{b}\right)=\frac{\beta_{b}-\sqrt{2 \alpha_{b}\left(U_{b}-u_{b}\left(w_{0}^{b}, w_{T}^{b}\right)\right)}}{\alpha_{b}} .
$$

\section{Concluding Remarks}

In this study, we considered Pareto efficiency in financial markets. The contribution of this study is to provide a framework to explicitly describe social welfare through the utility possibility frontier. In particular, we proposed the utility possibility frontier with the mean-variance utility. Moreover, in this study, the payoff function remains general. This enables us to examine various asset classes such as derivatives and insurance, and not simply basic securities. For example, we consider a simple insurance contract with default. We then demonstrate that the likelihood of default makes the market Pareto inefficient or deteriorates social welfare. 
Since it does not allow an agent with positive exposure to hedge the default risk, our market model is incomplete. On the other hand, the introduction of hedging methods, such as collateralization, might improve Pareto efficiency or social welfare. Such analysis is left for future studies.

\section{Supported}

This work was supported by JSPS KAKENHI Grant Number 17K18219.

\section{Conflicts of Interest}

The author declares no conflicts of interest regarding the publication of this paper.

\section{References}

[1] Acharya, V. and Bisin, A. (2014) Counterparty Risk Externality: Centralized versus Over-the-Counter Markets. Journal of Economic Theory, 149, 153-182. https://doi.org/10.1016/j.jet.2013.07.001

[2] Brennan, M.J. and Cao, H.H. (1996) Information, Trade, and Derivative Securities. The Review of Financial Studies, 9, 163-208. https://doi.org/10.1093/rfs/9.1.163

[3] Geanakoplos, J. (1997) Promises Promises, The Economy As An Evolving Complex System II. Taylor \& Francis Group LLC, Boca Raton, FL, 285-320. https://doi.org/10.1201/9780429496639-12

[4] Lioui, A. and Poncet, P. (2005) General Equilibrium Pricing of CPI Derivatives. Journal of Banking and Finance, 29, 1265-1294. https://doi.org/10.1016/j.jbankfin.2004.04.005

[5] Taddei, F. (2007) Collateral, Financial Arrangements and Pareto Optimality. Carlo Alberto Notebooks, 64, Collegio Carlo Alberto.

[6] Varian, H.R. (1992) Microeconomics Analysis. 3rd Edition, W.W. Norton \& Company, Inc., New York.

[7] Bessembinder, H. and Lemmon, M.L. (2002) Equilibrium Pricing and Optimal Hedging in Electricity Forward Markets. The Journal of Finance, 57, 1347-1382. https://doi.org/10.1111/1540-6261.00463

[8] Huh, Y. and Infante, S. (2016) Bond Market Intermediation and the Role of Repo, Working Paper. https://doi.org/10.2139/ssrn.2773678 https://ssrn.com/abstract $=2773678$

[9] Takino, K. (2016) An Equilibrium Model for the OTC Derivatives Market with A Collateral Agreement. Journal of Commodity Markets, 4, 41-55. https://doi.org/10.1016/j.jcomm.2016.11.001

[10] Munk, C. (2013) Financial Asset Pricing Theory. Oxford University Press, Oxford. 